\title{
A Non-Linear Model for Corrosion Fatigue Lifetime Based on Continuum Damage Mechanics
}

\author{
Jie Zhang ${ }^{1,2}$, Stijn Hertelé ${ }^{2}$, and Wim De Waele ${ }^{1}$ \\ ${ }^{1}$ SIM vzw, Technologiepark 935, BE-9052 Zwijnaarde, Belgium \\ ${ }^{2}$ Soete Laboratory, Department of EEMMeCS, Ghent University, Ghent, Belgium
}

\begin{abstract}
Many structures, such as oil platforms and wind turbines, are built in the marine environment. These structures do not merely suffer from variable cyclic loading due to wind, waves and current, but also from corrosion. Their interaction can give rise to corrosion fatigue, thus reducing the service life and integrity of the structure. The research community witnesses a challenge to identify damage mechanisms of combined fatigue loading and corrosion, and to link this to the lifetime prediction of offshore structures. In this paper, a non-linear corrosion fatigue model is proposed to describe damage accumulation based on continuum damage mechanics. Fatigue endurance limit, load frequency and corrosion rate are included in the model as basic parameters which influence the interaction between fatigue and corrosion. A sequential load effect is revealed via integration of the non-linear accumulation of damage. Parametric studies are conducted to show the abilities of the model. Preliminary simulation results agree to a strong extent with experimental data in the form of corrosion fatigue S-N curves. Nonetheless, deviations are observed at lower life times and these are prone to further investigation. In the future, parameters will be calibrated and further validation experiments will be performed.
\end{abstract}

\section{Introduction}

Offshore structures, such as supporting structures of offshore renewable energy applications, experience millions of cyclic loads due to wind, waves and current. Fatigue failure can occur when accumulated damage (propagation of an initiated fatigue crack) reaches the critical tolerance value of the structures. Offshore structures are also exposed to harsh environmental conditions during their operational life (1). The synergistic interaction between a corrosive environment and cyclic loading may amplify the development of fatigue damage, and is referred to as corrosion fatigue. As a result, materials deteriorate more severely $(2,3)$ which - if unaccounted for - may lead to catastrophes involving loss of lives. Therefore, it is important to develop a suitable model to predict the lifetime of the structure, and to instruct inspection and maintenance properly and timely.

It is reported that pits easily form and grow on the surface of materials in a corrosive environment (4-6). When this happens, changes in the surface morphology introduce stress concentrations which facilitate fatigue crack initiation. Therefore, dependent on the time and degree of corrosion, the formation of pits leads to a significant reduction of the fatigue resistance of metals $(7,8)$. However, it is difficult to satisfactorily model the entire physical mechanism due to the complexity of the corrosion fatigue process, which includes the stage of pit formation, pit growth, fatigue crack initiation and its propagation.

This study proposes a new model, by extending CDM approach by combining evolution model of fatigue limit. The effect of corrosion in translated into its associated influence on surface roughness. Damage of corrosion fatigue is discretized into fatigue damage in different pre-corroded conditions. The capability of mode to predict load interaction sequences effects are showed by a two-level loading study.

\section{Theoretical model}

The corrosion fatigue model proposed in this study is based on non-linear continuum damage mechanics (CDM), which is suitable for making engineering predictions about the initiation, propagation, and fracture of materials without resorting to a microscopic description that would be too complex for practical engineering analysis (9). The CDM method was first introduced by Kachanov (10) based on thermodynamic principle and. The proximity to local failure is defined as a cumulative damage variable. The rate of damage increase is based on state variables, which can be measured by macroscopic properties, Material degradation laws represent the effects of damage on the stiffness and remaining life of the material that is damaging as a result of thermomechanical load and ageing (11). CDM approaches describing fatigue are based on the generic equation shown below:

\footnotetext{
Corresponding author: author@e-mail.org
} 


$$
d D=f\left(\sigma_{\text {mean }}, \sigma_{a}, D, M C\right) d n
$$

(2-1)where

$d D$ is the fatigue damage increment per cycle $d n, \sigma_{a}$ and $\sigma_{\text {mean }}$ are alternating stress and mean stress, respectively; $\mathrm{MC}$ is material property constant.

For uniaxial loading, Chaboche et al. (12)proposed the following damage rate equation for high cycle fatigue:

$$
d D=\left[1-(1-D)^{1+\beta}\right]^{\alpha}\left[\frac{\sigma_{a}}{M(1-D)}\right]^{\beta} d n
$$

(2-2)where $\beta$ is a fixed material parameter representing, and $M$ and $\alpha$ are functions themselves of applied load parameters. Concretely, $M$ describes the dependency between mean stress and the fatigue limit. A linear dependency in terms of stress amplitude is commonly used:

$$
M\left(\sigma_{\text {mean }}\right)=M_{0}\left(1-b \sigma_{\text {mean }}\right)
$$

with, $M_{0}$ and $b$ additional material parameters. Then, $\alpha$ describes and is expressed as follows:

$$
\alpha=1-\frac{1}{H}\left\langle\frac{\sigma_{a}-\sigma_{f}\left(1-b \sigma_{\text {mean }}\right)}{\sigma_{u}-\sigma_{a}}\right\rangle
$$

(2-4)Where the symbol $<>$ represents the Heaviside step function $(<\mathrm{x}>=\mathrm{x}$ if $\mathrm{x}>0$ and $<\mathrm{x}>=0$ if $\mathrm{x}<0) ; \sigma_{u}$ and $\sigma_{f}$ are ultimate tensile stress and fatigue limit; $\mathrm{H}$ is a material constant. Local failure of material (associated with crack initiation at that point) is often assumed to occur when $D=1$. However, since CDM models like the above are often calibrated on the basis of S-N curves which express the total lifetime of a material (including crack initiation and propagation), researchers have also assumed crack initiation to occur at a critical damage value $D_{c}$ smaller than 1. In such case, $D_{c}$ is to be considered as an additional model parameter.

For the case of multiaxial loading, Chaudonneret(13) extended Chaboche work and proposed the multiaxial fatigue model for proportional and non-proportional loadings.

$$
\begin{aligned}
& d D=\left[1-(1-D)^{1+\beta}\right]^{\alpha\left(\sigma_{\text {mean }}, A_{H}\right)}\left[\frac{A_{I I}}{M_{0}\left(1-3 b \sigma_{H, \text { mean }}\right)(1-D)}\right]^{\beta} d n \\
& \quad \alpha=1-\frac{1}{H}\left\langle\frac{A_{I I}-\sigma_{f}\left(1-3 b \sigma_{H, \text { mean }}\right)}{\sigma_{u}-A_{I I}}\right\rangle
\end{aligned}
$$

(2-6)Where $A_{I I}$ is the amplitude of the octahedral shear stress, and $\sigma_{H \text {,mean }}$ is the mean value of hydrostatic pressure during the cycle. $A_{I I}$ and $\sigma_{H \text {,mean }}$ can be expressed as follows:

$$
\begin{gathered}
A_{I I}=\frac{1}{2} \sqrt{\frac{3}{2}\left(\sigma_{i j, \text { max }}-\sigma_{i j, \text { min }}\right)^{2}} \\
\sigma_{\text {mean }}^{H}=\frac{1}{6}\left[\sigma_{k k, \text { max }}+\sigma_{k k, \text { min }}\right]
\end{gathered}
$$

Where $\sigma_{i j, \max }$ and $\sigma_{i j, \min }$ are the maximum and minimum stress values of $\mathrm{ij}$ component of the deviator during a cycle respectively.

The basic idea of the model proposed in this study is to discretize fatigue damage into different corrosion conditions. For reasons of simplicity, the $D_{c}$ value equals to 1 in this study. The corrosion effect is represented by a time dependent reduction of the fatigue limit. Notably, ultimate tensile strength, elongation and elastic modulus of engineering metals (steels, aluminium and magnesium alloy) insignificantly decrease when immersed in a certain corrosive environment for different time span (14-16). Therefore, the ultimate tensile strength is assumed as a constant value in the proposed model. The effect of corrosion on fatigue properties, on the other hand, is far more influential. It can be translated into S-N curves obtained from pre-corroded specimens as described in related researches (17-20). A timedependent interpolation between S-N curves in absence and presence of different degrees of corrosion then allows to describe the coupled effect of corrosion and fatigue.

In the proposed model, the reduction of fatigue limit due to corrosion is expressed in terms of the associated increase in roughness. The latter can be expressed in various ways, such as the amplitude parameters $R_{a}$ and $R_{q}$, height parameters $R_{\rho}$ and $R_{z}$, spacing parameters $H_{S c}$ and $S_{m}$ and hybrid parameters $\Delta_{q}$ and $\lambda_{q}(21)$. We adopt $R_{a}$ as a roughness characteristic in the model. $R_{a}$ is the arithmetic average of the absolute values of the profile height deviations from the mean line, recorded within a certain evaluation length (22), as shown in Eq. ):

$$
R_{a}=\frac{1}{n} \sum_{i=1}^{n}\left|y_{i}\right| \quad R_{a}=\frac{1}{L} \int_{0}^{L}|Y(x)| d x
$$

(2-9)where y is the depth (or peak) from the mean line (see Figure 1, right). Therefore, the maximum depth of a corrosion pit serves as an upper bound of roughness $R_{a}$. In general terms, fatigue limit is expressed in terms of roughness and a certain reference roughness, as shown in Eq. (2-10):

$$
\sigma_{f}^{*}=f\left(R_{a}, \sigma_{f}\right)
$$

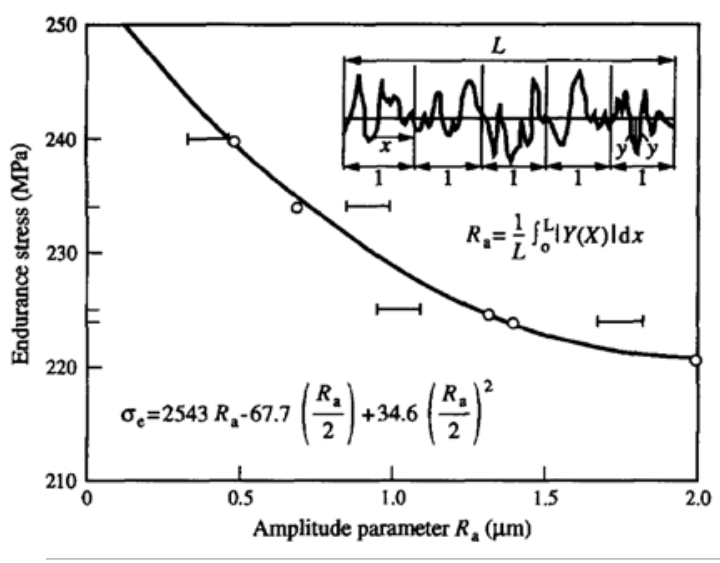




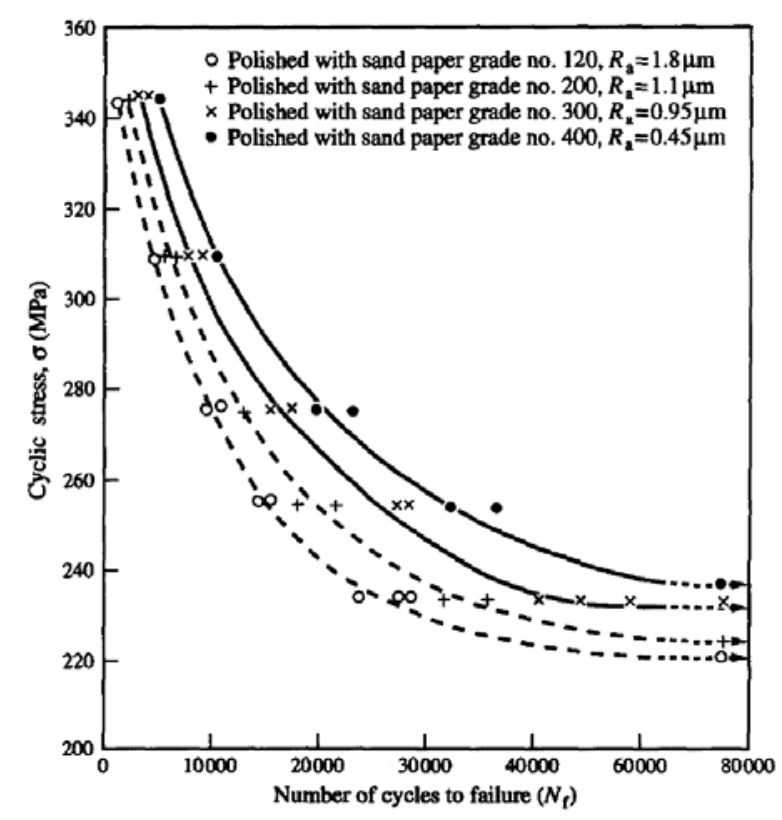

Figure 1 effect of surface roughness on number of cycles to failure(left) and effect of $R_{a}$ on fatigue limit(right) (21)

The development of corrosion pit size depends on electrochemical conditions. Based on abundant studies data collected in $(23,24)$, the time evolution of pit depth follows a power law, as shown in Eq.):

$$
d=g t_{(2-11)}^{h}
$$

where $\mathrm{d}$ is the depth of the corrosion pit; $\mathrm{t}$ is time; $\mathrm{g}$ and $\mathrm{h}$ are constants which are entirely dependent on material and electrochemical parameters.

According to Rybalka`s study(25), the depth of growing pit on $20 \mathrm{Kh} 13$ steel in $0.01 \mathrm{~m} \mathrm{NaCl}$ solution can be described as a function of time by $h \sim t^{0.5}$. A similar observation was made by Zhang et al. (26), who summarized various test data and plotted pit depth versus the square root of time, as shown in Figure 2. Cavanaugh(27) used Weibull function and neural network method characterized the pit depth and found its kinetics followed a $t^{1 / 3}$ relation. Sriraman(28) similarly proposes that depth is considered proportional to the cube root of time by $d=B t^{1 / 3}$.

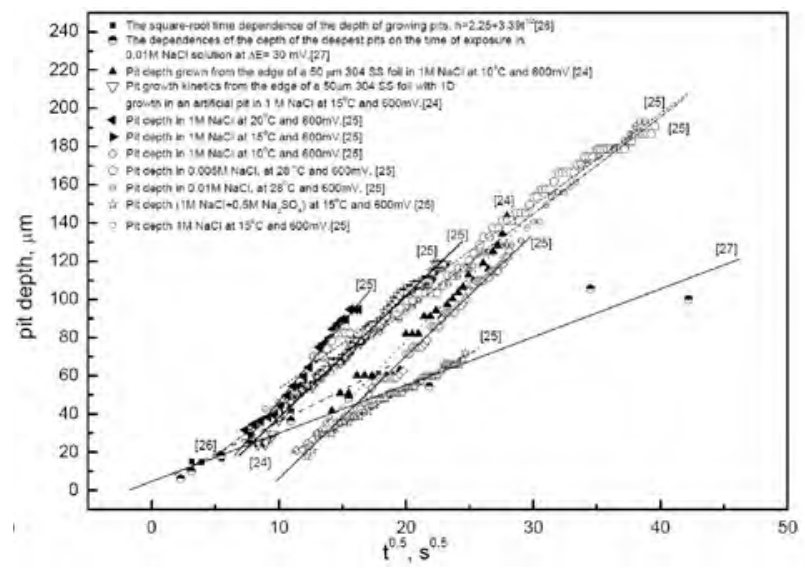

Figure 2 corrosion pit depth vs $t^{0.5}(26)$

According to Eqs. (2-9) to (2-11) it can be concluded that the evolution of fatigue limit in corrosion environment can be a function of time and parameters from material and corrosion.

$\sigma_{f}^{*}=F\left(\sigma_{f}, t, \mathrm{MC}\right)$

To couple the effect of corrosion with fatigue, $t$ can be linked to the number of fatigue cycles by means of the cycle frequency $f$, namely $t=n / f$.

Substituting fatigue limit $\sigma_{f}$ in damage model by $\sigma_{f}^{*}$, the corrosion fatigue damage rate can be expressed as follows:

$$
\frac{d D_{c f}}{d n}=\operatorname{power}\left[1-\left(1-D_{c f}\right)^{1+\beta}, 1-\frac{1}{H}\left\langle\frac{A_{I I}-\sigma_{f}^{*}\left(1-3 b \sigma_{H, \text { maan }}\right)}{\sigma_{u}-A_{I I}}\right\rangle\right]\left[\frac{A_{I I}}{M_{0}\left(1-3 b \sigma_{H, \text { mean }}\right)\left(1-D_{c f}\right)}\right]^{\beta}
$$

The model proposed in this paper contains a few parameters which are material properties due complexities in different physical contexts, and those parameters con be calibrated via matrix of experiments.

\section{Results and discussion}

Based on a hardnend and tempered steel, $30 \mathrm{NiCoMoV12}$, reported in literature (29), where an S-N curve is provided with fatigue limit equal to $391 \mathrm{MPa}$. This curve could be reasonably approximated using the basic Chaboche model, when adopting the following model parameters:

$$
\alpha=1-12.48\left\langle\frac{\sigma_{a}-391}{1035-\sigma_{a}}\right\rangle^{0.434}
$$

Then, an example of virtual corrosion effect was applied by implementing the model proposed in this study. The hypothetical data for relationship between time and roughness, roughness and fatigue limit are listed in Table 1 .

Table 1 virtual data of corrosion time, roughness and fatigue limit

\begin{tabular}{|c|c|c|c|c|}
\hline $\begin{array}{c}\text { corrosion } \\
\text { time/min }\end{array}$ & 0 & 6 & 12 & 18 \\
\hline $\begin{array}{c}\text { surface } \\
\text { roughness/ } \mu \mathrm{m}\end{array}$ & 0.45 & 0.7 & 1.1 & 1.8 \\
\hline $\begin{array}{c}\text { fatigue } \\
\text { limit/MPa }\end{array}$ & 391 & 250 & 160 & 50 \\
\hline
\end{tabular}

In Figure 3, blue and black curves are corrosion fatigue $\mathrm{SN}$ curves predicted by the model proposed in frequency $1 \mathrm{~Hz}$ and $10 \mathrm{~Hz}$ respectively. Comparing to the red curve, the two predicted curves indicate a reduced fatigue resistance, accelerated initiation at higher stresses and elimination of the fatigue limit. Generally, the results agree with the trend observed experimentally 
(30). Additionally, the model successfully predicts how a lower loading frequency leads to a lower fatigue resistance, as more corrosion damage develops during each cycle.

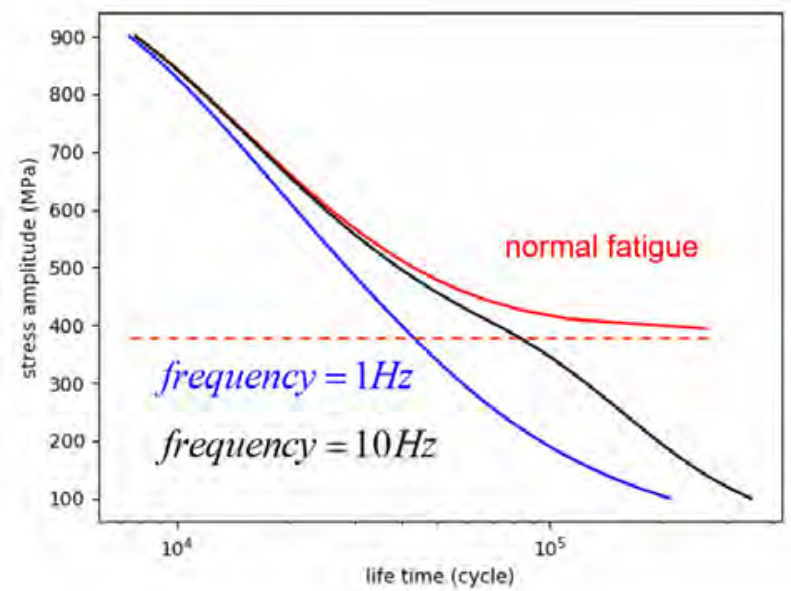

Figure 3 corrosion fatigue $\mathrm{SN}$ curves predicted in different load frequency in same corrosion condition

This model reveals non-linearity instinctively through differential equation of damage evolution rate, it can describe and predict load interaction sequence effect in the multi-level loading conditions. Two load levels at 450MPa and 550MPa have been applied as high-low and low-high sequence. The predicted data are plotted in Figure 4, which indicates the non-linearity of damage accumulation by the model proposed. By plotting two loads switching at damage value equal to 0.6 , Figure 5 illustrates one case of predicted evolution of corrosion fatigue damage accumulation by proposed model. The left in Figure 5 shows the total life is shortened comparably when the load sequence starting from low to high, vice versa, the total life is prolonged when load sequence is from high to low.

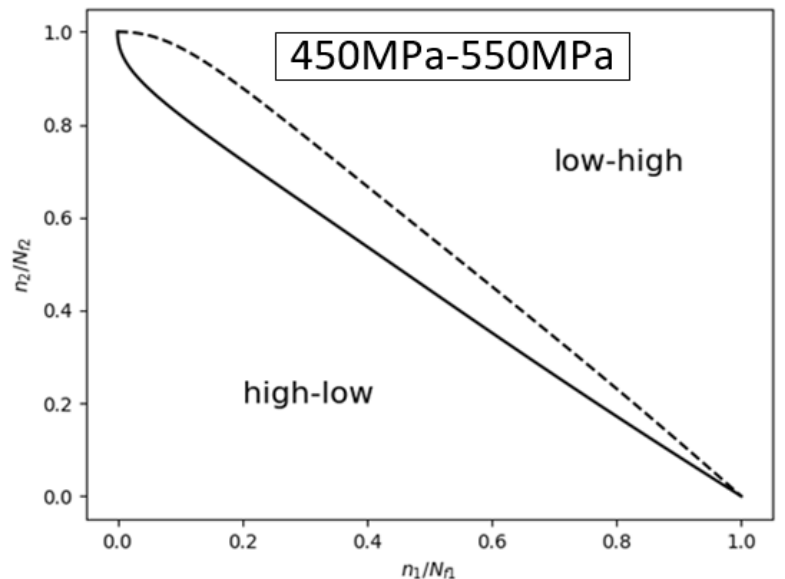

Figure 4 Prediction of sequence effect of two-level loading
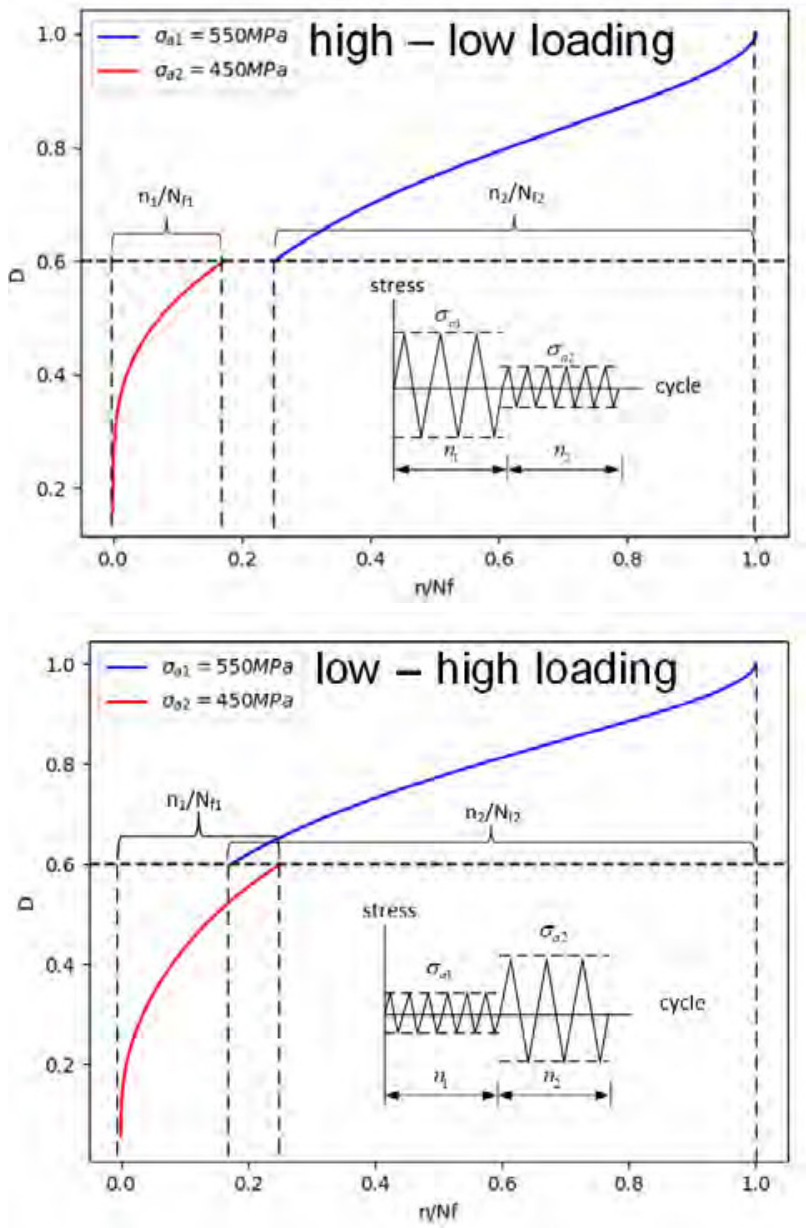

Figure 5 An example of predicted evolution of damage accumulation in high-low and low-high load sequence

\section{Conclusions}

This study proposes an innovative corrosion fatigue model based on non-linear continuum damage mechanics, which includes the corrosion effect by a time dependent degradation of the fatigue resistance curve. The numerical simulation shows that the model is capable to predict the corrosion effect and load sequence effect, and it successfully describes an interaction between corrosion and fatigue. This paper has focused on the methodology description and a study of the model capabilities. A validation study is currently being carried out, to evaluate the predictive ability of the model based on well-chosen calibration tests.

The authors gratefully acknowledge the financial support via MaDurOS program from SIM Flanders (Strategic Initiative Materials) and Vlaio (Flemish Agency for innovation and Entrepreneurship).

\section{References}

1. Adedipe O, Brennan F, Kolios Renewable and Sustainable Energy Reviews. 2016;

2. Marcus P. Corrosion Mechanisms in Theory and Practice, Third Edition: Taylor \& Francis; 2011. 
3. Magnin $T$, Delafosse D. CORROSION TECHNOLOGY-NEW YORK AND BASEL-. 2002;17

4. Rajasankar J, Iyer NR.. 2006;73(5)

5. Rokhlin SI, Kim JY, Nagy H, Zoofan B. 1999;62(4)

6. Dolley, Lee, Wei. 2000;23(7)

7. Havn T, Maritime A, Osvoll H. 2002/1/1/. NACE: NACE International.

8. Wang QY, Pidaparti RM, Palakal MJ. AIAA Journal. 2001;39(2)

9. Ebara R. Materials Science and Engineering: A. 2007;468-470

10. Krajcinovic D. Damage mechanics. Mechanics of Materials. 1989;8(2-3).

11. Kachanov LM. Rupture time under creep conditions. International journal of fracture. 1999;97(1-4)

12. Struik LCE. TU Delft, Delft University of Technology; 1977.

13. Chaboche JL, Lesne PM. Fatigue \& Fracture of Engineering Materials \& Structures. 1988;11(1)

14. Chaudonneret M. Journal of Engineering Materials and Technology. 1993;115(4)

15. Fu S, Gao H, Chen G, Gao L, Chen X. Materials Science and Engineering: A. 2014;593

16. Obert B, Ngo K, Hashemi J, Ekwaro-Osire S, Sivam TP. Journal of Materials Engineering and Performance. 2000;9(4)

17. Hou Y, Lei D, Li S, Yang W, Li C-Q. International Journal of Corrosion. 2016

18. $\mathrm{Hu} \mathrm{W}$, Shen Q, Zhang M, Meng Q, Zhang X. International Journal of Damage Mechanics. 2012;21(8)

19. Huang $\mathrm{Y}, \mathrm{Ye} \mathrm{X}, \mathrm{Hu} \mathrm{B}$, Chen $\mathrm{L}$. International Journal of Fatigue. 2016

20. Abdulstaar M, Mhaede M, Wagner L.. Advanced Engineering Materials. 2013;15(10)

21. Wang Z-b, Liu W-t, He X-f. Chinese Journal of Aeronautics. 2004;17(4)

22. Bayoumi MR, Abdellatif AK. 1995;51(5)

23. Institute ANS, Engineers ASoM. American Society of Mechanical Engineers; 2009

24. Harlow DG, Wei RP. Engineering Fracture Mechanics. 1998;59(3)

25. Kondo Y. CORROSION. 1989;45(1)

26. Rybalka KV, Beketaeva LA, Shaldaev VS, Kasparova LV, Davydov AD.. Russian Journal of Electrochemistry. 2009;45(11)

27. Zhang X-Y, Li S-X, Liang R, editors. ICF13; 2013.

28. Cavanaugh MK, Buchheit RG, Birbilis N. Corrosion Science. 2010;52(9)

29. Sriraman MR, Pidaparti RM. Journal of Aircraft. 2009;46(4)

30. Dattoma V, Giancane S, Nobile R, Panella FW. International Journal of Fatigue. 2006;28(2)

31. Devereux OF, McEvily AJ, Staehle RW, The University of Connecticut, Storrs, Connecticut: National Association of Corrosion Engineers; 1972. 